\title{
Comparison of regional flortaucipir PET with quantitative tau immunohistochemistry in three subjects with Alzheimer's disease pathology: a clinicopathological study
}

Michael J. Pontecorvo ${ }^{1 *}$ (D, C. Dirk Keene ${ }^{2}$, Thomas G. Beach ${ }^{3}$, Thomas J. Montine ${ }^{4}$, Anupa K. Arora', Michael D. Devous Sr${ }^{1}$, Michael Navitsky ${ }^{1}$, lan Kennedy ${ }^{1}$, Abhinay D. Joshi ${ }^{1,5}$, Ming Lu' ${ }^{1}$, Geidy E. Serrano ${ }^{3}$, Lucia I. Sue ${ }^{3}$, Anthony J. Intorcia ${ }^{3}$, Shannon E. Rose ${ }^{2}$, Angela Wilson², Leanne Hellstern², Natalie Coleman², Matthew Flitter ${ }^{1}$, Patricia Aldea ${ }^{1}$, Adam S. Fleisher', Mark A. Mintun ${ }^{1}$ and Andrew Siderowf ${ }^{1,6}$

\begin{abstract}
Background: The objective of this study was to make a quantitative comparison of flortaucipir PET retention with pathological tau and $\beta$-amyloid across a range of brain regions at autopsy.

Methods: Patients with dementia (two with clinical diagnosis of $A D$, one undetermined), nearing the end of life, underwent 20-min PET, beginning 80 min after an injection of $\sim 370 \mathrm{mBq}$ flortaucipir [ $\left.{ }^{18} \mathrm{~F}\right]$. Neocortical, basal ganglia, and limbic tissue samples were obtained bilaterally from 19 regions at autopsy and subject-specific PET regions of interest corresponding to the 19 sampled target tissue regions in each hemisphere were hand drawn on the PET images. SUVr values were calculated for each region using a cerebellar reference region. Abnormally phosphorylated tau (Ptau) and amyloid- $\beta$ (A $\beta$ ) tissue concentrations were measured for each tissue region with an antibody capture assay (Histelide) using AT8 and H31L21 antibodies respectively.

Results: The imaging-to-autopsy interval ranged from 4-29 days. All three subjects had intermediate to high levels of AD neuropathologic change at autopsy. Mean cortical SUVr averaged across all three subjects correlated significantly with the Ptau immunoassay (Pearson $r=0.81 ; p<0.0001$ ). When Ptau and $A \beta_{1-42}$ were both included in the model, the Ptau correlation with flortaucipir SUVr was preserved but there was no correlation of $A \beta_{1-42}$ with flortaucipir. There was also a modest correlation between limbic (hippocampal/entorhinal and amygdala) flortaucipir SUVr and Ptau (Pearson $r=0.52 ; p<0.080$ ). There was no significant correlation between SUVr and Ptau in basal ganglia.

\footnotetext{
* Correspondence: pontecorvo@avidrp.com

1'Avid Radiopharmaceuticals, 3711 Market St., 7th floor, Philadelphia, PA

19104, USA

Full list of author information is available at the end of the article
}

\section{Springer Open}

(c) The Author(s). 2020 Open Access This article is licensed under a Creative Commons Attribution 4.0 International License, which permits use, sharing, adaptation, distribution and reproduction in any medium or format, as long as you give appropriate credit to the original author(s) and the source, provide a link to the Creative Commons licence, and indicate if changes were made. The images or other third party material in this article are included in the article's Creative Commons licence, unless indicated otherwise in a credit line to the material. If material is not included in the article's Creative Commons licence and your intended use is not permitted by statutory regulation or exceeds the permitted use, you will need to obtain permission directly from the copyright holder. To view a copy of this licence, visit http://creativecommons.org/licenses/by/4.0/. 


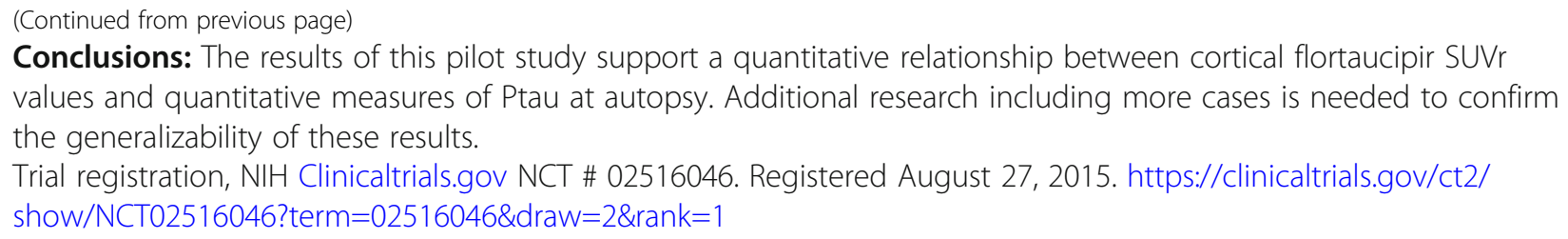

\section{Background}

The neuropathological hallmarks of Alzheimer's disease (AD) are extracellular accumulation of aggregated amyloid- $\beta(A \beta)$ peptides (plaques), often associated with degenerating neurites (neuritic plaques), and neurofibrillary degeneration characterized by intraneuronal aggregates of abnormally phosphorylated microtubuleassociated tau protein (Ptau) in paired helical filaments (PHF), historically termed neurofibrillary tangles (NFTs). In autopsy-verified disease, widespread deposition of tau, consistent with high Braak stage, is typically associated with cognitive impairment and is rarely seen in the absence of moderate to frequent neuritic plaques [1-6]. Based on these results, it has been speculated that $A \beta$ and Ptau changes may begin independently $[4,7]$, but $\mathrm{A} \beta$ plaques may be a necessary condition for the neocortical spread of NFTs, and consequent neuronal damage and cognitive impairment in $\mathrm{AD}$ [3].

Positron emission tomography (PET) imaging agents targeting $A \beta$ and Ptau have facilitated the exploration of these relationships in living persons. Florbetapir $\left[{ }^{18} \mathrm{~F}\right]$, florbetaben $\left[{ }^{18} \mathrm{~F}\right]$, and flutemetamol $\left[{ }^{18} \mathrm{~F}\right]$ are now approved for use in PET imaging of cerebral amyloid based on studies showing high sensitivity and specificity for predicting moderate to frequent neuritic plaques at autopsy [8-10].

More recently, tracers targeting pathologic tau have been reported [11-20]. The topographical pattern of tau tracer retention on PET imaging appears to roughly follow the neuropathological (autopsy) pattern of NFT accumulation [21], in that tau PET tracer retention is usually limited to mesial temporal lobe in clinically normal subjects, but appears to involve the lateral temporal, parietal and frontal lobes in amyloid-positive subjects with increasing degrees of cognitive impairment [2228]. Despite these encouraging initial results, it remains to be demonstrated that the pattern of abnormal tau signal on PET imaging of individual AD subjects matches the pattern of Ptau deposition at autopsy. To date, the most well-studied agent has been flortaucipir (flortaucipir $\left[{ }^{18} \mathrm{~F}\right]$, also known as flortaucipir $\mathrm{F} 18,\left[{ }^{18} \mathrm{~F}\right]-\mathrm{AV}-1451$ and $\left.\left[{ }^{18} \mathrm{~F}\right]-\mathrm{T} 807\right)$, but published imaging-to-autopsy studies with flortaucipir have predominantly evaluated nonAD dementias [29-35]. As expected from the established neuropathology of these conditions, cortical flortaucipir
PET signal in these subjects has generally been lower than what would be expected from typical AD patients $[33,35]$ and, where present, has tended to be greatest in anterior temporal lobes, frontal lobe, and striatum/globus pallidus [30, 31, 33, 34], as well as white matter [30]. Pathological evaluation ranged from semiquantitative scoring to quantitative immunohistochemistry (\% area occupied by hyperphosphorylated tau by Ptau staining) and the correlation between flortaucipir PET signal and the estimate of NFT/Ptau burden at autopsy ranged from not significant $[30,31,34]$ to excellent $(r=0.77-$ $0.93)[29,32,33]$. In contrast, a strong correlation between flortaucipir SUVr and tissue AT8 staining was recently reported for a patient with autosomal dominant (PSEN1) AD [36].

The objective of this study was to make a quantitative comparison of premortem flortaucipir PET retention and Ptau observed at autopsy, across multiple brain regions with varying densities of pathological tau, in autopsy-verified AD patients, using a robustly quantitative approach to determine regional protein concentration (Histelide technique) [37].

\section{Methods \\ Study design}

Two clinically diagnosed AD patients and one patient with dementia of unknown origin, near their end-of-life, were recruited for this pilot phase of a pivotal clinical trial (NCT \# 02516046). Participants had an injection of $\sim 370 \mathrm{mBq}$ flortaucipir $\left[{ }^{18} \mathrm{~F}\right]$ followed by PET imaging from 80-100 min after injection. After death, bilaterally symmetrical neocortical and limbic blocks of tissue were dissected from 19 limbic and neocortical regions as described below. Subject-specific PET regions of interest corresponding to pathology blocks were constructed for the 19 target regions above as well as the cerebellar cortex in each hemisphere. Regions were hand drawn on the PET images using dimension-scaled photographs of the gross pathology blocks as reference. Standard uptake value ratios (SUVr) were calculated using a cerebellar reference region. Ptau and $A \beta$ were assessed with a quantitative antibody capture assay, using the AT8 and $\mathrm{A} \beta_{1-42}$ antibodies, respectively. Regional quantitative 
estimates of flortaucipir PET signal and Ptau density were compared using regression/correlation statistics.

This protocol was approved by the relevant institutional review boards and all subjects or authorized representatives signed informed consent prior to the conduct of study procedures. This trial was conducted in compliance with the Declaration of Helsinki and the International Conference on Harmonization guidelines on good clinical practice.

\section{Imaging acquisition and analysis}

For flortaucipir PET imaging, subjects received an IV infusion of approximately $370 \mathrm{MBq}(10 \mathrm{mCi})$ of flortaucipir $\left[{ }^{18} \mathrm{~F}\right]$, followed approximately $80 \mathrm{~min}$ post-dose, by a 20-min brain scan, acquired as four 5-min frames. All PET data were reconstructed with an iterative or rowaction maximum likelihood algorithm with a $128 \times 128$ or $200 \times 200$ image matrix, pixel size of $2-2.67 \mathrm{~mm} \times$ $2-2.67 \mathrm{~mm}$, slice thickness of $2-4.25 \mathrm{~mm}$, and postreconstruction Gaussian filter of 3-5 mm or a relaxation parameter of normal or sharp filter.

Two experienced readers visually interpreted flortaucipir images as having either an AD pattern (TAD: involving, in moderate cases, increased neocortical flortaucipir activity in posterior lateral temporal lobe or, in advanced cases, in the parietal/precuneus regions or in the frontal region in addition to posterior-lateral temporal, parietal, or occipital) or not-AD pattern (TAD-: No increased neocortical activity, or increased neocortical activity isolated to the mesial temporal, anterolateral temporal, and/or frontal regions).

For the quantitative analysis of flortaucipir PET data, the 5-min PET images were summed into a single 20min image and regions of interest (ROI) were hand drawn by an experienced reader in native space to match the regions used for histopathological sampling (below). Matching was done by comparing $\mathrm{x}, \mathrm{y}$, and $\mathrm{z}$ axis dimensions as displayed on the photographed brain sections and scaling the location of sampled sections to the PET dimensions. Once scaled, each ROI was drawn to match as closely as possible the anatomic section location (see example in Supplemental Figure 1). Flortaucipir PET signal was expressed as SUVr values at the regional level, by dividing the PET signal in the target area by the average signal within the cerebellar cortex.

\section{Neuropathology}

All neuropathological protocols were performed with appropriate consent and in accordance with applicable regulations and Institutional Review Boards. Brains of subjects were collected at the respective study sites, fixed in $10 \%$ neutral buffered formalin for approximately 3 weeks, and shipped to the central study site for processing. Once fixed, brains were coronally sectioned into approximately $1 \mathrm{~cm}$ thick slices and samples taken for paraffin processing and embedding using standard cassettes. Tissue samples were processed and embedded in paraffin wax according to standard protocols, sectioned at $5 \mu \mathrm{m}$ thickness, and mounted on standard charged glass slides for histology or Histelide assays. Levels of Alzheimer's disease neuropathological change (ADNC), including Braak neurofibrillary stages, were assigned according to National Institute on Aging-Alzheimer's Association consensus guidelines [38, 39], using immunohistochemical methods for Ptau (AT8 antibody: MN1020, ThermoFisher Scientific) and A $\beta$ (6E10, Covance) pathology, as well as the Bielschowsky silver method for neuritic plaques. As part of the standard neuropathology evaluation, slides were also assessed on a regional basis for the presence of glial or astrocytic (atypical) tau pathology.

Tissue samples/slides prepared for the Histelide analysis included the all the neocortical and limbic regions from each hemisphere required for Braak staging as part of a standard AD neuropathologic diagnosis, specifically hippocampus/entorhinal cortex, amygdala, middle frontal gyrus, superior and middle temporal gyrus, inferior parietal lobe, and Brodmann's area (BA) 17 and BA 18 in the occipital lobe. Additional regions sampled bilaterally included basal ganglia at the level of the anterior commissure/nucleus basalis, thalamus, anterior cingulate, posterior temporal lobe BA 37, inferior lateral temporal (BA 20, 21), parietal occipital junction (BA 39), precuneus (BA 7), frontal premotor (BA 6), anterior frontal (BA 9), orbital frontal (BA 11), primary motor cortex (BA 4), and basal ganglia at the level of the full development of the lentiform nucleus (caudate, putamen, and globus pallidus).

\section{Histelide quantitative antibody capture assay}

The Histelide assay was performed as previously described [37]. Briefly, formalin-fixed paraffin-embedded brain tissue from study participants was mounted on charged microscope slides at $5 \mu \mathrm{m}$ thick then deparaffinized and rehydrated using xylenes and isopropanol. Tissue slides were incubated in the primary antibody for $2 \mathrm{~h}$ at room temperature. AT8 (MN1020, ThermoFisher Scientific) and H31L21 A $\beta$ (700254, ThermoFisher Scientific) primary antibodies were used to assess Ptau and amyloid pathology, respectively. Primary antibody exposure was followed by alkaline-phosphatase conjugated secondary antibody incubation overnight at room temperature. Tissue slides were incubated in $\mathrm{p}$ nitrophenyl phosphate (pNPP) solution for $90 \mathrm{~min}$ and the absorbance of p-nitrophenyl (pNP), the chromogenic product of (pNPP)-alkaline phosphatase reaction, was measured by spectrophotometer. Analytical grade pNP dilution standards were used to create a 4-parameter 
equation calibration curve (fitted using Spectromax M2 software) to convert absorbance readings to pNP $(\mu \mathrm{g})$, a surrogate measure of the antibody-bound protein-ofinterest in the sample. pNP mass was normalized to total tissue area (i.e., $\mu \mathrm{g} / \mathrm{cm}^{2}$ ), measured using a light microscope and Stereo Investigator software (MBF Bioscience). Non-specific background was subtracted using adjacent tissue slide incubated with IgG isotype control primary antibody, and three internal standard controls were included across all runs to control for batch effects. Additional details are found in the supplemental material.

\section{Statistical methods}

Demographics and subjects' characteristics, including clinical diagnosis and cognitive assessment by MiniMental State Examination (MMSE) were collected. Considering the small sample size, and the exploratory nature of this analysis, a simple Pearson's correlation analysis was applied to investigate the relationship between pathological and imaging findings. To further explore the potential clustering effect by subject, a partial correlation was run by conditioning on the subject. An additional partial correlation analysis was run by conditioning both by subject and the other pathological measurement. For example, when investigating the relationship between flortaucipir SUVr and quantitative ptau immunohistochemistry, the correlation analysis was adjusted for both subject and amyloid assessment.

\section{Results}

Three participants with a clinical diagnosis of dementia (two clinically diagnosed AD patients and one patient with dementia of unknown origin), with an anticipated life expectancy of less than 6 months at entry, were included in the pilot phase of the study. The intervals between imaging and death for the three cases were 4, 10, and 29 days. Patient characteristics are provided in Table 1. The flortaucipir PET images demonstrating antemortem evidence of pathologic tau in each patient are shown in Fig. 1a. The three subjects varied in degree and distribution of flortaucipir retention on the PET scans. Case 2 showed the least retention, limited primarily to the mesial and lateral temporal lobe. Case 3 showed the most intense PET signal encompassing virtually all of the lateral temporal, occipital, and parietal lobes but with largely rightlateralized activity in the frontal lobe. Case 1 did not have as intense a PET signal as Case 3, but flortaucipir retention was more evident in the frontal lobes, as well as in lateral temporal and parietal lobes. All three cases were visually interpreted as having an AD pattern of pathologic tau (TAD) on flortaucipir PET.

At consensus neuropathological diagnosis $(38,39)$, Braak stage by hemisphere ranged from IV to VI, but each subject was at least Braak V (B3) in at least one hemisphere. Case 1 had the highest Braak stage score (R/L:VI/V) and Case 2 the lowest (R/L:IV/V). Representative AT8 stained slides are shown in Fig. 1b. All three patients had moderate to frequent CERAD neuritic plaque densities. Cases 1 and 3 had an amyloid Thal phase of 4 (A3), and thus met the criteria for high $\mathrm{AD}$ neuropathological change [38]. Thal phase in Case 2 was 3 ; thus, this case met the criteria for intermediate $A D$ neuropathological change. Atypical Ptau pathology (e.g., glial or morphologically non-AD neuronal tau) was not noted for any subject.

There was a highly significant correlation across all patients between neocortical regional flortaucipir SUVr and the Histelide estimate of Ptau total tissue concentration (Fig. 2, blue symbols; Pearson $r=0.81 ; p<0.0001$;

Table 1 Characteristics of patients followed to autopsy.

\begin{tabular}{llll}
\hline & Case 1 & Case 2 & Case 3 \\
\hline Age & 77 & 86 & 86 \\
Gender & Male & Female & Male \\
Race & White & White & 8 \\
Years of Symptoms & 4 & 2 & NA \\
MMSE & NA & 20 & AD \\
Clinical diagnosis & AD & Dementia Unknown Origin & 29 \\
Scan to autopsy ${ }^{1}$ interval (days) & 4 & 10 & 2 \\
Death to autopsy interval (days) & 0 & 0 & 4 \\
Thal stage & 4 & 3 & VN \\
Braak stage (R,L) & VIN & IVN & Frequent \\
CERAD neuritic plaques & Frequent & Moderate & Absent \\
Atypical tau pathology & Absent & Absent & \\
\hline
\end{tabular}

Abbreviations: $A D$ Alzheimer's dementia, $L$ left, $N A$ not assessed, $R$ right

${ }^{1}$ Date of autopsy defined as the start of the autopsy, i.e., date of brain tissue collection 

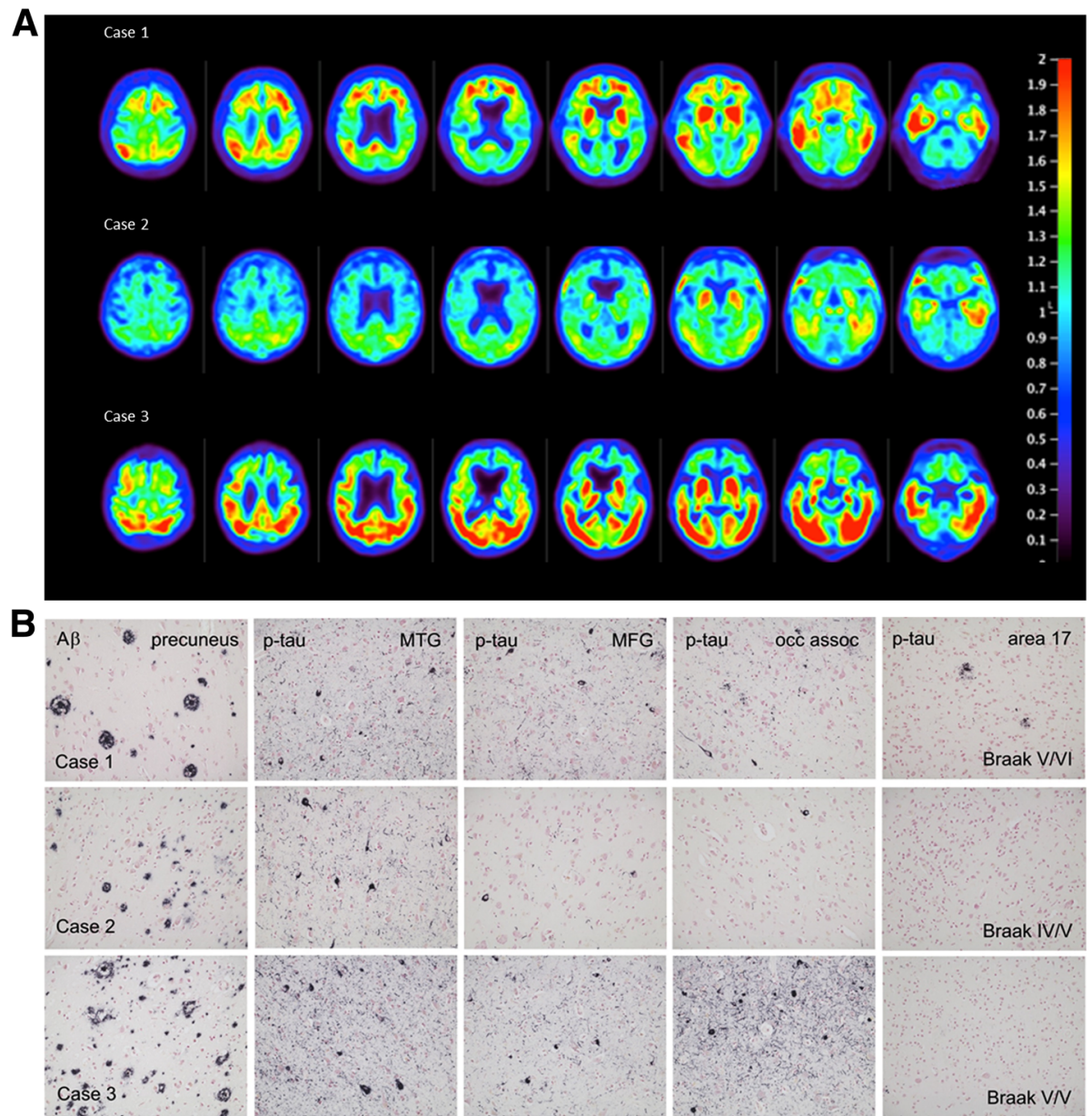

Fig. 1 a Flortaucipir PET images: premortem evaluation of tau. Flortaucipir PET images acquired 80-100 min post 370 MBq iv administration. Voxel-wise SUVr values are represented relative to a cerebellar reference region and scaled for a range of 0-2.0. b Representative slides showing amyloid (6E10 antibody) and regional Ptau (AT8, MN1020 antibody) pathology in the three cases. MTG: middle temporal gyrus; MFG: middle frontal gyrus; occ assoc: occipital lobe peristriate association cortex, Brodmann area 18

Table 2, Model 1). Although the range of values differed across individuals, similar trends were seen within individual subjects (Supplemental Figure 2). Correcting the regression model for individual cases did not meaningfully alter the $r$-value (Pearson $r=0.81 ; p<0.0001$; Table 2, Model 2). There was also a modest correlation between limbic (hippocampal/entorhinal and amygdala) flortaucipir SUVr and the Histelide estimate of Ptau total tissue concentration (Fig. 2, green symbols; Pearson $r=$ 0.52; $p=0.080$ ). However, the slope of the SUVr/Ptau relationship appeared perhaps steeper for these limbic regions than for the cortical regions, suggesting a possibly reduced sensitivity of flortaucipir SUVr to Ptau in these limbic regions. There was no correlation between flortaucipir SUVr and the Histelide estimates for subcortical regions including thalamus, ventromedial globus pallidus, and caudate-putamen (Fig. 2, red symbols; Pearson $r=0.27 ; p=0.280$ ).
Table 2 shows that there was also an apparent correlation between neocortical flortaucipir SUVr and the Histelide estimate of $\mathrm{A} \beta$ total tissue concentration (Table 2, row 2: Pearson $r=0.61 ; p<0.001)$. However, this is likely due to colinearity between Ptau and A $\beta$ (Pearson $r$ $=0.66 ; p<0.0001$ ), as significance for the flortaucipir SUVr- A $\beta$ correlation did not survive adjustment for Ptau tissue concentration in multivariable models (Table 2, Model 3, Pearson $r=0.01, p=0.9302$ ). In contrast, the correlation between flortaucipir PET SUVr and Histelide estimate of Ptau total tissue concentration survived correction when $\mathrm{A} \beta$ was included in the model (Pearson $r=0.73, p<0.0001$ ).

\section{Discussion}

The results showed significant associations between neocortical flortaucipir PET signal (SUVr values) and Ptau tissue concentrations across regions among subjects with 


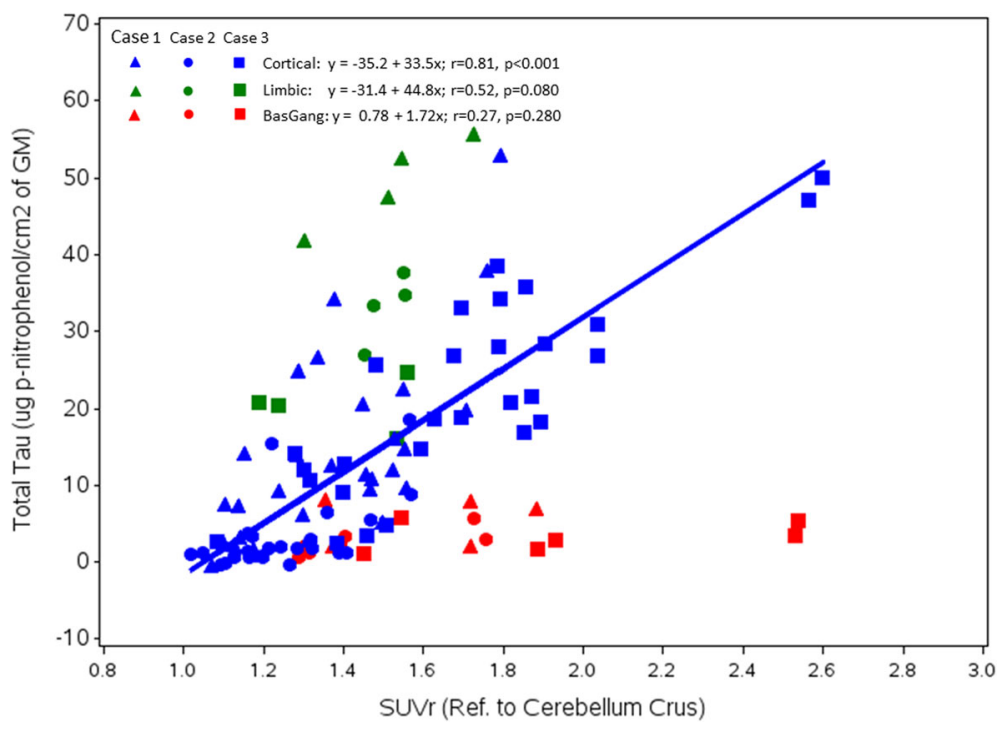

Fig. 2 Associations shown between regional flortaucipir SUVr and Ptau total tissue concentration (Histelide) values at autopsy. Regression line shows the relationship between cortical regional SUVr and cortical regional Ptau. Cortical regions are shown in blue, limbic in green, and basal ganglia in red. Symbols differentiate individual subjects

different Braak stages, and importantly, within subjects but across different brain regions. Our findings are consistent with the widely accepted descriptions of the density and extent of neurofibrillary degeneration in $\mathrm{AD}$ in the medical literature $[4,21,38-40]$ and provide initial evidence that flortaucipir PET may be an accurate measure of quantitative differences in the density of the Ptau that is most consistently associated with severity of cognitive impairment in $\mathrm{AD}$ [4-6].

There was also a correlation between limbic (hippocampal/entorhinal and amygdala) flortaucipir SUVr and the Histelide estimate of Ptau tissue concentration. However, the slope of the relationship appeared steeper than for neocortical measures, suggesting a possibly reduced sensitivity of flortaucipir SUVr to Ptau in these limbic regions. It is not clear whether this is due to artifact of PET signal loss subsequent to atrophy and the resultant partial volume effects on the PET scans [24] or whether this reflects a lower affinity of flortaucipir for the types of pathologic tau found in the mesial temporal lobe of patients with AD [41].

In contrast to neocortical and limbic areas, there was no correlation between flortaucipir PET signal and
Histelide estimate of Ptau in the subcortical regions examined (striatum, globus pallidus, nucleus basalis, and thalamus). The lack of correlation of flortaucipir signal with striatum Ptau is consistent with previous studies that have shown presumably "off-target" flortaucipir PET signal in these regions even in cognitively normal elderly subjects that would be expected to have little or no Ptau $[22,26,42]$. The differing kinetics of flortaucipir PET in these regions [42] suggests the presence of a different, lower affinity, high-density binding site that is probably not related to NFTs.

Several clinicopathological case reports have investigated the relationship between flortaucipir imaging and pathological changes in patients with non-AD tauopathies or amyloidoses. One such study showed no selective flortaucipir retention (minimal retention similar to cognitively normal controls) and no increased occurrence of NFTs in subjects with autopsy-confirmed Creutzfeldt-Jakob disease [35]. In a patient with autopsyconfirmed corticobasal degeneration (CBD), the greatest densities of pathological tau and the highest PET SUVrs were found, as might be predicted, in subcortical regions including the putamen and pallidum [29]. Another

Table 2 Correlations among cortical flortaucipir SUVr and Histelide estimates of Ptau and A $\beta$ tissue concentrations at autopsy

\begin{tabular}{|c|c|c|c|c|c|c|}
\hline & \multicolumn{2}{|c|}{ Model 1 (no adjustment) } & \multicolumn{2}{|c|}{ Model 2 (adjusted for case) } & \multicolumn{2}{|c|}{ Model 3 (adjusted for case and other pathology } \\
\hline & Pearson $\boldsymbol{r}$ & $\boldsymbol{p}$ Value & Pearson $\boldsymbol{r}$ & $\boldsymbol{p}$ Value & Pearson $\boldsymbol{r}$ & $p$ Value \\
\hline Ptau vs SUVr & 0.81 & $p<0.0001$ & 0.81 & $p<0.0001$ & 0.73 & $p<0.0001$ \\
\hline$A \beta$ vs SUVr & 0.61 & $p<0.0001$ & 0.52 & $p<0.0001$ & 0.01 & $p=0.9302$ \\
\hline Ptau vs $A \beta$ & 0.66 & $p<0.0001$ & & & & \\
\hline
\end{tabular}

Model 1: No adjustment; Model 2: partial correlation by adjusting for case ID; Model 3: partial correlation by adjusting for case ID, and the other pathology. For example, the correlation between PHF tau and SUVr was adjusted for case ID and A $\beta_{1-42}$ 
study, again in a patient with confirmed CBD, showed similar results [32]. A fourth case report, in a patient with autopsy-confirmed Parkinson's disease, found elevated flortaucipir PET signal in areas previously associated with off-target flortaucipir retention (basal ganglia, midbrain, choroid plexus) and in the inferior temporal cortex, the latter consistent with the evidence of $\mathrm{AD}$ like, possibly age-related, NFT accumulation in the entorhinal cortex at autopsy [31]. The same group found similar results in two progressive supranuclear palsy (PSP) patients and a MAPT P301L carrier [30], with the greatest retention observed on flortaucipir PET in basal ganglion and midbrain, and only low levels of retention in cortical areas; mainly frontal, anterior temporal, and white matter regions. At autopsy, Ptau immunoreactive inclusions in these cases were more widespread and abundant than predicted by the imaging results. However, with the exception of the entorhinal cortex, the Ptau pathology in such subjects is morphologically and biochemically different (e.g., differences in the relative amounts of $3 R$ and $4 R$ tau) from that seen in $A D$. In contrast, a series of 3 patients with frontotemporal dementia due to a MAPT R406W mutation (which produces $3 R / 4 R$ tau aggregates similar to those in AD) showed highly significant correlations between flortaucipir PET and neuropathological grading and semi-quantitative assessment of tau-positive neurites [33]. Finally, a case report of a living patient with familial frontotemporal dementia due to a MAPT $10+$ $16 \mathrm{C}>\mathrm{T}$ gene mutation showed a significant association between tau imaging and the familial pattern of pathology [43]. One interpretation of this conflicting data is that there is the heterogeneity of flortaucipir binding in non-AD tauopathies and that flortaucipir is more likely to recognize pathology from patients with MAPT mutations that produce more AD-like tau pathology.

Consistent with this hypothesis, a strong correlation between regional flortaucipir SUVr and percent area labeled by AT8 at autopsy was reported for an autosomal dominant PSEN-1 AD subject. Although flortaucipir SUVr values in the present sporadic AD patients were considerably lower than the SUVr in the autosomal dominant case, a similar relationship was observed in the present study between regional SUVr and regional Ptau, as estimated from AT8-Histelide. Thus, our results contribute to existing evidence of a concordance between imaging and AD pathological change.

We also found a modest correlation between the density of $A \beta$ and Ptau, as separately measured by Histelide, consistent with a previous report [44]. This colinearity was most likely responsible for the apparent correlation between flortaucipir PET SUVr and $A \beta_{1-42}$ tissue concentrations at autopsy. When Ptau was included in the model, the significance of its correlation with flortaucipir SUVr was preserved while that with $A \beta_{1-42}$ was not.

Hyperphosphorylated tau pathology may occur in both glial cells and neurons in both AD and non-AD dementias. In this respect, it is important to note both that the subjects in this study all had sufficient amyloid and Ptau pathology to meet the National Institute of Aging-Alzheimer's Association criteria for intermediate or high ADNC [38]. However, abnormal tau pathology (e.g., glial tau) was not identified in these cases at autopsy. Thus, it seems unlikely that non-AD glial or neuronal tau pathology is accounting for the observed flortaucipir PET signal.

Alternatively, it has been suggested $[45,46]$ that flortaucipir binds to monoamine oxidase (MAO). However, it is not clear that the reported in vitro affinity of flortaucipir to MAO translates into in vivo signal. The pattern of retention of flortaucipir does not correspond to the known distribution of MAO activity in the brain (e.g., in contrast to MAO there is limited flortaucipir retention in the thalamus of normal subjects). Recently, it was demonstrated that the AD-tau-like cortical retention for another putative tau tracer, THK 5351, could be displaced by the administration of the MAO-B inhibitor selegeline [47]. It was suggested that rather than, or in addition to tau, THK 5351 might be binding to MAO-B in neuritic plaque-associated astrocytes, thus contributing both to the AD-tau-like pattern of retention. Studies in our lab do not support a high-affinity binding of flortaucipir to MAO-B [48], and patients taking MAO-B blockers do not show reduced retention of flortaucipir [49]. Thus, we believe the most parsimonious explanation for the association between flortaucipir PET and Ptau at autopsy is that flortaucipir binds to and reflects the density of Ptau in the brain, except for off-target regions where the basis of the binding remains unknown.

Our results must be considered in the context of some study limitations. Because of the fragile condition of the subjects (all within 30 days of death), MRI was not performed, which limits the precision with which the PET ROI can be matched to the histopathology blocks. Additionally, the Histelide values were derived from slides representing only a small portion of that block, whereas the PET ROIs, albeit drawn to match the histopathology blocks, represent a much larger area than an individual slide. SUVr values were calculated using a cerebellum reference region. The pathology expert panel did not evaluate NFT in the cerebellum, but Histelide estimates of phosphorylated protein levels in the cerebellum were less than or equal to estimates in other presumably low tau subcortical regions. Perhaps most important, this is a small case-series and only included subjects with clinically defined dementia, with autopsy evidence of intermediate to high ADNC (all three cases had at least 
Braak V NFT stage, and moderate to frequent neuritic plaques but Case 2 only had a Thal amyloid phase of 3 ). The range of flortaucipir SUVr and Histelide Ptau estimates varied among the three subjects (Supplemental Figure 1). Given the small sample size in this study, it is fair to conclude that additional subjects would be required to more precisely characterize the degree of relationship between flortaucipir PET signal and Ptau density, particularly with respect to limbic regions, where only six data points are available for evaluation.

A strength of the study is that PET regions of interest were drawn and PET scans interpreted without knowledge of quantitative histology results; histology was interpreted in a blinded fashion as well. Although it is possible that other approaches (e.g., mapping of anatomically derived standard regions to the PET scans) could have been taken, the current strategy has the further advantage of closely matching the location of the PET ROIs to the individual regions sampled for histopathological evaluation. Other strengths of this study include the standardized acquisition of PET data and the fact that brain samples came from two different clinical sites.

\section{Conclusion}

In conclusion, this study provides preliminary evidence that flortaucipir PET imaging captures the density and distribution of pathologic neocortical tau in a manner that is correlated with findings from a quantitative antibody capture assay. By extension, these findings suggest that flortaucipir PET signal may be useful in assessing neurofibrillary degeneration in $\mathrm{AD}$.

\section{Supplementary information}

Supplementary information accompanies this paper at https://doi.org/10. 1186/s13550-020-00653-X.

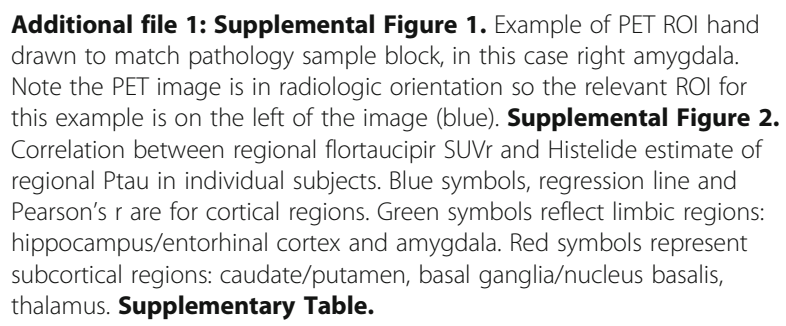

\section{Abbreviations}

AD: Alzheimer's disease; ADNC: Alzheimer's disease neuropathological change; $A \beta$ : Amyloid $\beta$; CBD: Corticobasal degeneration (corticobasal syndrome); BA: Brodmann area; MAO: Monoamine oxidase; MMSE: MiniMental State Examination; MRI: Magnetic resonance imaging; NFT: Neurofibrillary tangle; PET: Positron emission tomography; PHF: Paired helical filament tau; pNP: p-Nitrophenol; pNPP: p-Nitrophenol phosphate; PSP: Progressive supranuclear palsy; Ptau: Abnormally phosphorylated tau; ROI: Region of interest; SUVr: Standardized uptake value ratio

\section{Acknowledgements}

\section{Authors' contributions}

MJP, AKA, MDD, MAM, AS, TGB, TJM, and CDK contributed to conception and design. MJP, AKA, MDD, MAM, AS, PA, and MF contributed to acquisition and analysis and supervised the enrollment of patients and the collection of imaging data and autopsy tissue. TGB, TJM, CDK, GES, LIS, AJ, SER, AW, LH, and NC performed the neuropathological/Histelide analyses. MDD, MN, IK, and AJD performed the image quantitation. ML performed the statistical analyses. MJP, AKA, MDD, MAM, AS, TGB, TJM, CDK. MJP, MDD, AS, TGB, TJM, CDK, MN, IK, ML contributed to interpretation, drafting, and revision of the manuscript. All authors read and approved the final manuscript.

Funding

This study was sponsored by Avid Radiopharmaceuticals, a wholly owned subsidiary of Eli Lilly and Company.

\section{Availability of data and materials}

Flortaucipir SUVr and Ptau values shown in Fig. 2 and the supplemental figure are included in the supplemental materials.

\section{Ethics approval and consent to participate}

All procedures performed in studies involving human participants were in accordance with the ethical standards of the institutional and/or national research committee and with the 1964 Helsinki declaration and its later amendments or comparable ethical standards. This protocol was approved by the relevant institutional review boards and all subjects or authorized representatives signed informed consent prior to the conduct of study procedures.

It is not our policy to disclose information about enrollment at particular centers or to identify centers where a particular patient was enrolled, as this potentially could unmask patient identity. Providing detailed information about ethics committees could reveal the enrollment center and contribute to patient unmasking, particularly in a report such as this one where individual data on only three patients (as opposed to aggregate data) is reported. However, we can confirm that the overall A16 study (NCT \# 02516046) was reviewed and approved by a central IRB (Advarra, formerly Schulman Associates, IRB, 6940 Columbia Gateway Dr., Suite 110, Columbia, MD 21046).

\section{Consent for publication}

The consent form signed by each patient or representative included the statement: study data that does not directly identify you may be published in medical journals or shared with others as part of scientific discussions. A copy of our template consent form (required text for all sites) is available on request.

\section{Competing interests}

MJP, AKA, MDD, MN, IK, ML, PA, MF, ASF, and MAM are full-time employees of Avid Radiopharmaceuticals, a wholly owned subsidiary of Eli Lilly and Company, and are minor stockholders of Eli Lilly and Company. ADJ and AS were employees of Avid at the time this work was performed but are now at Medpace Holdings and University of Pennsylvania, respectively. TGB, TJM, CDK, GES, LIS, AJI, SER, AW, LH, and NC contributed to this work as part of a research contract with Avid.

\section{Author details}

${ }^{1}$ Avid Radiopharmaceuticals, 3711 Market St., 7th floor, Philadelphia, PA 19104, USA. 'Department of Pathology, University of Washington, Seattle, WA, USA. ${ }^{3}$ Civin Laboratory for Neuropathology, Banner Sun Health Research Institute, Phoenix, AZ, USA. ${ }^{4}$ Department of Pathology, Stanford University, Stanford, CA, USA. ${ }^{5}$ Present Address: Medpace Holdings, Inc., Cincinnati, Ohio, USA. ${ }^{6}$ Present Address: Department of Neurology, University of Pennsylvania, Philadelphia, PA, USA.

Received: 13 December 2019 Accepted: 3 June 2020

Published online: 15 June 2020

References

1. Bennett DA, Schneider JA, Bienias JL, Evans DA, Wilson RS. Mild cognitive impairment is related to Alzheimer's disease pathology and cerebral infarctions. Neurology. 2005;64:834-41. 
2. Knopman DS, Parisi JE, Salviati A, Floriach-Robert M, Boeve BF, Ivnik RJ, et al. Neuropathology of cognitively normal elderly. J Neuropathol Exp Neurol. 2003;62:1087-95.

3. Nelson PT, Jicha GA, Schmitt FA, Liu H, Davis DG, Mendiondo MS, et al. Clinicopathologic correlations in a large Alzheimer disease center autopsy cohort: neuritic plaques and neurofibrillary tangles "do count" when staging disease severity. J Neuropathol Exp Neurol. 2007;66:1136-46.

4. Nelson PT, Braak H, Markesbery WR. Neuropathology and cognitive impairment in Alzheimer disease: a complex but coherent relationship. J Neuropath Exp Neurol. 2009;68:1-14.

5. Nelson PT, Alafuzoff I, Bigio EH, Bouras C, Braak H, Cairns NJ, et al. Correlation of Alzheimer disease neuropathologic changes with cognitive status: a review of the literature. J Neuropathol Exp Neurol. 2012;71:362-81.

6. Tomlinson BE, Blessed G, Roth M. Observations on the brains of demented old people. J Neurol Sci. 1970;11:205-42.

7. Mungas D, Tractenberg R, Schneider JA, Crane PK, Bennett DA. A twoprocess model for neuropathology of Alzheimer's disease. Neurobiol Aging. 2014;35:301-8.

8. Clark CM, Pontecorvo MJ, Beach TG, Bedell BJ, Coleman RE, Doraiswamy PM, et al. Cerebral PET with florbetapir compared with neuropathology at autopsy for detection of neuritic amyloid-beta plaques: a prospective cohort study. Lancet Neurol. 2012;11:669-78.

9. Curtis C, Gamez JE, Singh U, Sadowsky CH, Villena T, Sabbagh MN, et al. Phase 3 trial of flutemetamol labeled with radioactive fluorine 18 imaging and neuritic plaque density. JAMA Neurol. 2015;72:287-94.

10. Sabri O, Sabbagh MN, Seibyl J, Barthel H, Akatsu H, Ouchi Y, et al. Florbetaben PET imaging to detect amyloid beta plaques in Alzheimer's disease: phase 3 study. Alzheimers Dement. 2015;11:964-74.

11. Betthauser TJ, Cody KA, Zammit MD, Murali D, Converse AK, Barnhart TE et al. In vivo characterization and quantification of neurofibrillary tau PET radioligand MK-6240 in humans from Alzheimer's disease dementia to young controls. bioRxiv. March 28, 2018. https://doi.org/10.1101/290064.

12. Chien DT, Szardenings AK, Bahri S, Walsh JC, Mu F, Xia C, et al. Early clinical PET imaging results with the novel PHF-tau radioligand [F18]-T808. J Alzheimers Dis. 2014;38:171-84.

13. Declercq L, Rombouts F, Koole M, Fierens K, Mariën J, Langlois $X$, et al. Preclinical evaluation of ${ }^{18} \mathrm{~F}$-JNJ64349311, a novel pet tracer for tau imaging. J Nucl Med. 2017:58:975-81.

14. Harada R, Okamura N, Furumoto S, Furukawa K, Ishiki A, Tomita N, et al. ${ }^{18} \mathrm{~F}$ THK5351: a novel PET radiotracer for imaging neurofibrillary pathology in Alzheimer disease. J Nucl Med. 2016;57:208-14.

15. Maruyama M, Shimada H, Suhara T, Shinotoh H, Ji B, Maeda J, et al. Imaging of tau pathology in a tauopathy mouse model and in Alzheimer patients compared to normal controls. Neuron. 2013;79:1094-108.

16. Okamura N, Furumoto S, Harada R, Tago T, Yoshikawa T, Fodero-Tavoletti M, et al. Novel ${ }^{18} \mathrm{~F}$-labeled arylquinoline derivatives for noninvasive imaging of tau pathology in Alzheimer's disease. J Nuc Med. 2013;54:1420-7.

17. Saint-Aubert L, Lemoine L, Chiotis K, Leuzy A, Rodriguez-Vieitez E, Nordberg A. Tau PET imaging: present and future directions. Mol Neurodegener. February 20, 2017; 12: 19. https://doi.org/10.1186/s13024-017-0162-3.

18. Villemagne $V L$, Dore V, Bourgeat $P$, Burnham SC, Laws S, Salvado O, et al. $A \beta$-amyloid and tau imaging in dementia. Semin Nucl Med. 2017;47:75-88.

19. Wong DF, Comley R, Kuwabara H, Rosenberg PB, Resnick SM, Ostrowitzki S, et al. Characterization of 3 novel tau radiopharmaceuticals, ${ }^{11} \mathrm{C}-\mathrm{RO}-963,{ }^{11} \mathrm{C}$ $\mathrm{RO}-643$, and ${ }^{18} \mathrm{~F}-\mathrm{RO}-948$, in healthy controls and in Alzheimer subjects. J Nucl Med. 2018;59:1869-76.

20. Xia CF, Arteaga J, Chen G, Gangadharmath U, Gomez LF, Kasi D, et al. [18F]T807, a novel tau positron emission tomography imaging agent for Alzheimer's disease. Alzheimers Dement. 2013;9:666-76.

21. Braak H, Alafuzoff I, Arzberger T, Kretzschmar H, Del Tredici K. Staging of Alzheimer disease-associated neurofibrillary pathology using paraffin sections and immunocytochemistry. Acta Neuropathol. 2006;112:389-404.

22. Brier MR, Gordon B, Friedrichsen K, McCarthy J, Stern A, Christensen J, et al. Tau and $A B$ imaging, CSR measures, and cognition in Alzheimer's disease. Sci Transl Med. 2016;8:338 ra66. https://doi.org/10.1126/scitransImed.aaf2362.

23. Cho H, Choi JY, Hwang MS, Kim YJ, Lee HM, Lee HS, et al. In vivo cortical spreading pattern of tau and amyloid in the Alzheimer's disease spectrum. Ann Neurol. 2016;80:247-58.

24. Johnson KA, Schultz A, Betensky RA, Becker JA, Sepulcre J, Rentz D, et al. Tau positron emission tomographic imaging in aging and early Alzheimer disease. Ann Neurol. 2016;79:110-9.
25. Lockhart SN, Baker SL, Okamura N, Furukawa K, Ishiki A, Furumoto S, et al. Dynamic PET measures of tau accumulation in cognitively normal older adults and Alzheimer's disease patients measured using [18F] THK-5351. PLoS One. June 29, 2016; 11: e0158460. https://doi.org/10.1371/journal.pone. 0158460.

26. Pontecorvo MJ, Devous MD Sr, Navitsky M, Lu M, Salloway S, Schaerf FW, et al. Relationships between flortaucipir ( ${ }^{18} \mathrm{~F}-\mathrm{AV}$-1451) positron emission tomography tau binding and amyloid burden clinical diagnosis, age and cognition. Brain. 2017;140:748-63.

27. Schöll M, Lockhart SN, Schonhaut DR, O'Neil JP, Janabi M, Ossenkoppele R, et al. PET imaging of tau deposition in the aging human brain. Neuron. 2016;89:971-82.

28. Schwarz AJ, Yu P, Miller BB, Shcherbinin S, Dickson J, Navitsky M, et al. Regional profiles of the candidate tau PET ligand ${ }^{18} \mathrm{~F}-\mathrm{AV}$-1451 recapitulate key features of Braak histopathological stages. Brain. 2016;139:1539-50.

29. Josephs KA, Whitwell JL, Tacik P, Duffy JR, Senjem ML, Tosakulwong N, et al. [18F]AV-1451 tau-PET uptake does correlate with quantitatively measured $4 \mathrm{R}$-tau burden in autopsy confirmed corticobasal degeneration. Acta Neuropathol. 2016:132:931-3.

30. Marquié M, Normandin MD, Meltzer AC, Siao tick Chong M, Andrea NV, Antón-Fernández A, et al. Pathologic correlations of [F-18]-AV-1451 imaging in non-Alzheimer tauopathies. Ann Neurol. 2017;81:117-28.

31. Marquié M, Verwer EE, Meltzer AC, Kim SJW, Agüero C, Gonzalez J. Lessons learned about [F-18]-AV-1451 offtarget binding from an autopsy-confirmed Parkinson's case. Acta Neuropathol Comm. 2017;5:75. https://doi.org/10. 1186/s40478-017-0482-0

32. McMillan CT, Irwin DJ, Nasrallah I, Phillips JS, Spindler M, Rascovsky K, et al. Multimodal evaluation demonstrates in vivo ${ }^{18} \mathrm{~F}-\mathrm{AV}$-1451 uptake in autopsyconfirmed corticobasal degeneration. Acta Neuropathol. 2016;132:935-7.

33. Smith $R$, Puschmann $A$, Schöll $M$, Ohlsson $T$, van Swieten J, Honer $M$, et al, 18F-AV-1451 tau PET imaging correlates strongly with tau neuropathology in MAPT mutation carriers. Brain. 2016;139:2372-9.

34. Smith R, Scholl M, Honer M, Nilsson CF, Englund E, Hansson O. Tau neuropathology correlates with FDG-PET but not AV-1451-PET in progressive supranuclear palsy. Acta Neuropathol. 2017;133:149-51.

35. Day GS, Gordon BA, Perrin RJ, Cairns NJ, Beaumont H, Schwetye K, et al. In vivo $\left[{ }^{18} \mathrm{~F}\right]-\mathrm{AV}-1451$ tau-PET imaging in sporadic Creutzfeldt-Jakob disease. Neurology. Mar 6; 2018; 90(10):e896-e906. doi: https://doi.org/10.1212/WNL. 0000000000005064

36. Smith R, Wilbom M, Pawlik D, Englund E, Hansson O. Correlation of in vivo $\left[{ }^{18} \mathrm{~F}\right]$ flortaucipir with postmortem Alzheimer disease tau pathology. JAMA Neurol. 2019;76:310-7.

37. Postupna N, Rose SE, Bird TD, Gonzalez-Cuyar LF, Sonnen JA, Larson EB, et al. Novel antibody capture assay for paraffin-embedded tissue detects wide-ranging amyloid beta and paired helical filament-tau accumulation in cognitively normal older adults. Brain Pathol. 2012;22:472-84.

38. Hyman BT, Phelps CH, Beach TG, Bigio EH, Cairns NJ, Carrillo MC, et al. National Institute on Aging Alzheimer's Association guidelines for the neuropathologic assessment of Alzheimer's disease. Alzheimers Dement. 2012;8:1-13.

39. Montine TJ, Phelps CH, Beach TG, Bigio EH, Cairns NJ, Dickson DW, et al. National Institute on Aging - Alzheimer's Association guidelines for the neuropathologic assessment of Alzheimer's disease: a practical approach. Acta Neuropathol. 2012;123:1-11.

40. Braak H, Braak E. Neuropathological stageing of Alzheimer-related changes. Acta Neuropathol. 1991;82:239-59.

41. Lowe VJ, Curran G, Fang P, Liesinger AM, Josephs, KA, Parisi, JE, et al. An autoradiographic evaluation of AV-1451 tau PET in dementia. Acta Neuropathologica Commun. June 13, 2016; 4: 58. https://doi.org/10.1186/ s40478-016-0315-6.

42. Shcherbinin S, Schwarz AJ, Joshi AJ, Navitsky M, Flitter M, Shankle WR, et al. Kinetics of the tau PET tracer $\left[{ }^{18} \mathrm{~F}\right]-\mathrm{AV}-1451$ (T807) in subjects with normal cognitive function, mild cognitive impairment and Alzheimer's disease. J Nuc Med. 2016;10:1535-42.

43. Bevan Jones WR, Cope TE, Passamonti L, Fryer TD, Hong YT, Aigbirhio F, et al. [ ${ }^{18}$ FAV-1451] PET in behavioral variant frontal temporal dementia due to MAPT mutation. Ann Clin Trans Neurol. 2016;3:940-7.

44. Postupna N, Keene CD, Crane PK, Gonzalez-Cuyar LF, Sonnen JA, Hewitt J, et al. Cerebral cortical A 42 and PHF-t in 325 consecutive brain autopsies varies by diagnosis, location, and APOE. J Neuropathol Exp Neurol. 2015;74: 100-9. 
45. Marquié M, Normandin MD, Vanderburg CR, Costantino IM, Bien EA, Rycyna LG, et al. Validating novel tau positron emission tomography tracer [F-18]AV-1451 (T807) on postmortem brain tissue. Ann Neurol. 2015;78:787-800.

46. Vermeiren C, Motte P, Viot D, Mairet-Coello G, Courade JP, Citron M, et al. The tau positron-emission tomography tracer AV-1451 binds with similar affinities to tau fibrils and monoamine oxidases. Mov Disord. 2018;33:27381.

47. Ng KP, Pascoal TA, Mathotaarachchi S, Therriault J, Kang MS, Shin M, et al. Monoamine oxidase B inhibitor, selegiline, reduces ${ }^{18} \mathrm{~F}-$ THK5351 uptake in the human brain. Alzheimers Res Ther. 2017;9:25. https://doi.org/10.1186/ s13195-017-0253-y.

48. Wright J, Lin YG, Goodman J, Lieberman B, Clemens J, Hoye A, et al. Studies to evaluate the putative binding of the tau positron emission tomography tracer ${ }^{18} \mathrm{~F}-\mathrm{AV}-1451$ to monoamine oxidase-B (MAO-B). Alzheimer's association international conference, Los Angeles, CA 2019. Alzheimers Dementia. 2019;15:75-2 131.

49. Hansen AK, Brooks DJ, Borghammer P. MAO-B inhibitors do not block in vivo flortaucipir([(18)F]-AV-1451) binding. Mol Imaging Biol. 2018;20:35660.

\section{Publisher's Note}

Springer Nature remains neutral with regard to jurisdictional claims in published maps and institutional affiliations.

\section{Submit your manuscript to a SpringerOpen ${ }^{\circ}$ journal and benefit from:}

- Convenient online submission

- Rigorous peer review

- Open access: articles freely available online

High visibility within the field

- Retaining the copyright to your article

Submit your next manuscript at $\boldsymbol{\nabla}$ springeropen.com 